OPEN ACCESS

Edited by:

Minjie Lu,

Chinese Academy of Medical

Sciences and Peking Union Medical

College, China

Reviewed by:

Stefano Coiro,

Hospital of Santa Maria della

Misericordia in Perugia, Italy

Katarzyna Mizia-Stec,

Medical University of Silesia, Poland

${ }^{*}$ Correspondence:

Weichun Wu

achundoctor@163.com

Zhenhui Zhu

dr.zhu@139.com

Hao Wang

hal6112@163.com

Specialty section

This article was submitted to

Cardiovascular Imaging,

a section of the journal

Frontiers in Cardiovascular Medicine

Received: 30 September 2021

Accepted: 13 December 2021

Published: 17 January 2022

Citation:

Lin J, Gao L, He J, Liu M, Cai Y, Niu L,

Zhao Y, Li X, Wang J, Wu W, Zhu Z and Wang $H$ (2022) Comparison of Myocardial Layer-Specific Strain and

Global Myocardial Work Efficiency

During Treadmill Exercise Stress in

Detecting Significant Coronary Artery

Disease.

Front. Cardiovasc. Med. 8:786943.

doi: 10.3389/fcrm.2021.786943

\section{Comparison of Myocardial} Layer-Specific Strain and Global Myocardial Work Efficiency During Treadmill Exercise Stress in \section{Detecting Significant Coronary Artery Disease}

Jingru Lin ${ }^{1}$, Lijian Gao ${ }^{2}$, Jia He ${ }^{3}$, Mengyi Liu ${ }^{1}$, Yuqi Cai ${ }^{1}$, Lili Niu ${ }^{1}$, Ying Zhao ${ }^{1}$, Xiaoni Li ${ }^{1}$, Jiangtao Wang ${ }^{4}$, Weichun $\mathrm{Wu}^{1,5 *}$, Zhenhui Zhu ${ }^{1 *}$ and Hao Wang ${ }^{1 *}$

'Department of Echocardiography, National Center for Cardiovascular Diseases, Fuwai Hospital, Chinese Academy of Medical Sciences and Peking Union Medical College, Beijing, China, ${ }^{2}$ Department of Cardiology, National Center for Cardiovascular Diseases, Fuwai Hospital, Chinese Academy of Medical Sciences and Peking Union Medical College, Beijing, China, ${ }^{3}$ Cardiac Arrhythmia Center, National Center for Cardiovascular Diseases, Fuwai Hospital, Chinese Academy of Medical Sciences and Peking Union Medical College, Beijing, China, ${ }^{4}$ General Electric Healthcare, Beijing, China, ${ }^{5}$ Key Laboratory of Cardiovascular Imaging (Cultivation), Chinese Academy of Medical Sciences, Beijing, China

Background: Myocardial layer-specific strain can identify myocardial ischemia. Global myocardial work efficiency (GWE) based on non-invasive left ventricular (LV) pressure-strain loops is a novel parameter to determine LV function considering afterload. The study aimed to compare the diagnostic value of GWE and myocardial layer-specific strain during treadmill exercise stress testing to detect significant coronary artery disease (CAD) with normal baseline wall motion.

Methods: Eighty-nine patients who referred for coronary angiography due to suspected of $C A D$ were included. Forty patients with severe coronary artery stenosis were diagnosed with significant CAD, and 49 were defined as non-significant CAD. Stress echocardiography was performed $24 \mathrm{~h}$ before angiography. Layer-specific longitudinal strains were assessed from the endocardium, mid-myocardium, and epicardium by $2 \mathrm{D}$ speckle-tracking echocardiography. Binary logistic regression analyses were performed to evaluate the association between significant CAD and echocardiographic parameters. A receiver operating characteristic curve was used to assess the capability of layer-specific strain and GWE to diagnose significant CAD.

Results: Patients with significant CAD had the worse function in all three myocardial layers at peak exercise compared with those with non-significant CAD when assessed with global longitudinal strain (GLS). At the peak exercise and recovery periods, GWE was lower in patients with significant CAD than in patients with non-significant CAD. In multivariable binary logistic regression analysis, peak endocardial GLS (OR: 1.35, $p=0.006$ ) and peak GWE (OR: 0.76, $p=0.001$ ) were associated with significant CAD. Receiver operating characteristic curves showed peak GWE to be superior to mid-myocardial, epicardial, and endocardial GLS in identifying significant CAD. Further, 
adding peak GWE to endocardial GLS could improve diagnostic capabilities.

Conclusions: Both GWE and endocardial GLS contribute to improving the diagnostic performance of exercise stress echocardiography. Furthermore, adding peak GWE to peak endocardial GLS provides incremental diagnostic value during a non-invasive screening of significant CAD before radioactive or invasive examinations.

Keywords: myocardial work efficiency, layer-specific strain, speckle-tracking echocardiography, treadmill exercise stress, coronary artery disease

\section{INTRODUCTION}

Coronary artery disease $(\mathrm{CAD})$ is one of the leading global causes of mortality and morbidity. Concerning the diagnostics of CAD, coronary angiography (CAG), which despite being considered the gold standard, only confirmed significant CAD in $38 \%$ of suspected patients (1). Stress echocardiography, which is recommended as a first-line diagnostic test in patients with suspected of CAD, showed a limited specificity and sensitivity due to lacking quantitative and objective methods (2). Noninvasive, quantitative, and objective imaging techniques are necessary for an optimal decision on diagnosis and therapy in patients suspected of CAD without known heart disease to improve clinical outcomes and enhance the diagnostic yield of cardiac catheterization.

The newly developed and explored (3-5) quantitatively objective method of layer-specific strain evaluation could increase the diagnostic accuracy for CAD (5) by evaluating the endocardial myocardium's longitudinal function, which was more susceptible to ischemic injury.

However, as strain is load-dependent, increasing the afterload may underestimate the left ventricular (LV) function; therefore, myocardial work (MW), which combines strain and noninvasive LV pressure, could overcome the limitation. Global myocardial work efficiency (GWE) is one of the major MW parameters and is derived from the percentage ratio of constructive work to the sum of constructive work and wasted work. Recently, significantly lower GWE values were reported in patients with $\mathrm{CAD}$, heart failure, hypertension with left ventricle hypertrophy, and COVID-19 (6-10), and the prognostic value of GWE was found to predict the longterm outcomes of patients after ST-segment elevation myocardial infarction (11) or patients after cardiac resynchronization therapy (12).

Due to the limited data available, this study aims to compare the diagnostic value of GWE with myocardial layer-specific global longitudinal strain during exercise stress to detect significant CAD with normal baseline wall motion.

Abbreviations: CAD, coronary artery disease; 2D, two-dimensional; MW, myocardial work; LV, left ventricular; GWE, global myocardial work efficiency; GLS, global longitudinal strain; CAG, coronary angiography; ECG, electrocardiography; WMSI, wall motion score index; AFI, automated function imaging; AUC, area under the curve.

\section{MATERIALS AND METHODS}

\section{Study Population}

The study was conducted in a single tertiary coronary care center with 89 patients without known ischemic heart disease who were referred with angina pectoris and had plans to undergo treadmill exercise stress testing and CAG. Patients with the following criteria were excluded: age $<18$ years; baseline LV ejection fraction $<50 \%$; abnormal baseline wall motion; previous myocardial infarction; prior coronary artery bypass grafting or coronary interventional therapy; left bundle branch block; atrial fibrillation; sustained severe arrhythmia; severe valvular dysfunction; inability to undergo exercise testing; absence of any of apical four-chamber, three-chamber, and two-chamber views; and inadequate image quality. All patients underwent two-dimensional (2D) echocardiography (speckle-tracking echocardiography and MW analysis), and a treadmill exercise stress test followed by CAG.

Written informed consent was given by all study participants or their legal representatives. The study complied with the Declaration of Helsinki and was approved by the Ethics Committees of Fuwai Hospital (no. 2018-1121).

\section{Conventional Echocardiography}

Comprehensive conventional echocardiography was performed (13) using a commercially available ultrasound system (Vivid E9 or Vivid E95, GE Healthcare, Horton, Norway) with a 3.5$\mathrm{MHz}$ transducer. Images were analyzed offline using dedicated software (EchoPAC 203, GE Healthcare, Horton, Norway). LV dimensions, LV septal thickness, and LV posterior wall thickness were measured from the parasternal long-axis view. $\mathrm{LV}$ mass was calculated as $\{0.8 \times 1.04 \times[(\mathrm{LV}$ end-diastolic diameter + LV end-diastolic posterior wall thickness + enddiastolic septal wall thickness $\left.)^{3}-(\text { LV end-diastolic diameter })^{3}\right]$ +0.6 . LV mass was then indexed for body surface area to generate the LV mass index. LV ejection fraction was measured using Simpson's biplane method from apical fourand two-chamber views. Early trans-mitral velocity (E wave) and late trans-mitral velocity (A wave) were measured by pulsed-wave Doppler from the apical four-chamber view with the sample volume positioned at the tip of the mitral leaflets. Mitral inflow E/A ratio was calculated as $\mathrm{E}$ wave divided by A wave. 


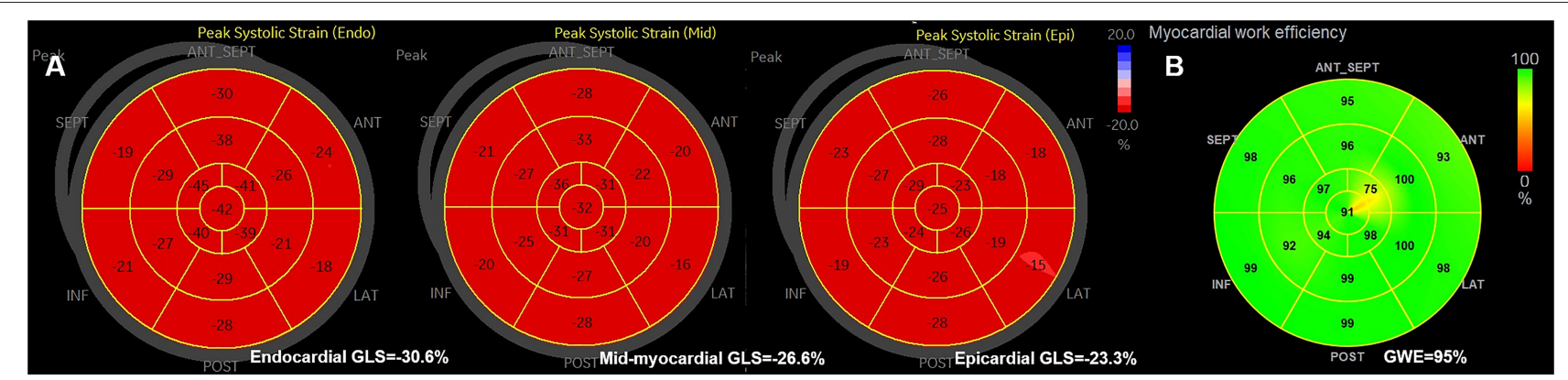

FIGURE 1 | (A) The example of bull's eyes of the global myocardial longitudinal layer-specific strain. (B) The example of global myocardial work efficiency. GLS, global longitudinal strain; GWE, global myocardial work efficiency.

\section{Treadmill Exercise Stress Echocardiography}

The treadmill exercise stress test was conducted utilizing the standard Bruce protocol (14) whereby the patient's heart rate, blood pressure, and 12-lead electrocardiography (ECG) were recorded. Cine loops (2D) from the four-, two-, and threechamber apical views were taken during the rest (before exercise), peak exercise ( $<1 \mathrm{~min}$ after exercise), and recovery ( $3 \mathrm{~min}$ after exercise) periods. Criteria for terminating the test were achieving a target heart rate of $85 \%$ of the age-predicted maximum, development of wall motion abnormality, development of intolerant symptoms, severe ischemic electrocardiographic changes, severe hypertension (systolic blood pressure $>220$ $\mathrm{mmHg}$ or diastolic blood pressure $>120 \mathrm{mmHg}$ ), symptomatic hypotension, or significant arrhythmia (14). The ECGs were categorized as either normal or abnormal by two blinded investigators. A positive exercise ECG was defined as STsegment horizontal or down-sloping depression $\geq 1 \mathrm{~mm}$ with duration $>2$ min or ST-segment elevation $\geq 1 \mathrm{~mm}$ with duration $>1 \mathrm{~min}$ in the leads dominated by $\mathrm{R}$ waves. The regional myocardial functional abnormality was assessed based on the observed wall thickening and endocardial motion of the myocardial segment in exercise stress echocardiography (15). A 17-segment model was used to assess wall motion from the apical four-, two-, and three-chamber views during the rest, peak exertion, and recovery periods, and a semiquantitative scoring system was used to analyze each segment $(1=$ normal wall motion, $2=$ hypokinesia, $3=$ akinesia, and $4=$ dyskinesia) $(15,16)$. The wall motion score index (WMSI) was calculated for each patient as the average of the analyzed segmental values, and patients with wall motion abnormality (WMSI > 1) during exercise were considered as positive stress echocardiogram.

\section{Speckle-Tracking Echocardiography and Myocardial Layer-Specific Strain}

From each of the three apical views at rest and during exercise, the cardiac loop with the best representation of the LV wall was identified and analyzed in the commercially available software (EchoPAC version 203, GE Healthcare); initially with automated function imaging (AFI) and subsequently with the 2D strain software. AFI identified and tracked the endocardium automatically to generate the LV global longitudinal strain (GLS), and the regional speckle area of interest was adjusted manually where the tracking was poor. For 2D strain analysis, the endocardial borders were manually delineated by the operator and then traced by the software in the end-systolic frame for the analyses of longitudinal endocardial, midmyocardial, and epicardial strains (Figure 1). Images with low tracking quality in more than two segments in a single view were excluded from further analysis. The $\Delta$-value of the LV myocardial layer-specific measurements was calculated as the difference between the values at peak exercise stress and at rest.

\section{Global LV Myocardial Work Efficiency Analysis}

Global myocardial work efficiency was calculated from a combination of the 2D LV GLS (based on AFI) and a non-invasively estimated LV pressure, using the commercially obtained software (EchoPAC version 203, GE Healthcare) (17). Brachial blood pressure was measured immediately after the echocardiography test, as a surrogate of non-invasive peak LV pressure. A non-invasive pressure-strain loop was constructed from the software adjusted to the timing of valvular events, mitral valve closure, aortic valve opening, aortic valve closure, and mitral valve opening (18). Myocardial work index corresponds to the area of the pressure-strain loop during the cardiac cycle. Myocardial constructive work was defined as work performed by the myocardium during segmental shortening in systole or during lengthening in isovolumic relaxation. Myocardial wasted work was defined as work performed by the myocardium during segmental lengthening in systole or during segmental shortening against a closed aortic valve in isovolumic relaxation. GWE was calculated as the sum of constructive work in all LV segments, divided by the sum of constructive and wasted work in all LV segments, expressed as a percentage (Figure 1). 
TABLE 1 | Clinical characteristics and conventional echocardiographic variables of patients.

\begin{tabular}{|c|c|c|c|}
\hline Variable & $\begin{array}{l}\text { Non-Significant } \\
\text { CAD }(n=49)\end{array}$ & $\begin{array}{l}\text { Significant CAD } \\
(n=40)\end{array}$ & $p$-value \\
\hline Age, years & $56 \pm 8$ & $58 \pm 8$ & 0.202 \\
\hline Male, $n(\%)$ & $30(61)$ & $31(78)$ & 0.100 \\
\hline Body surface area, $\mathrm{m}^{2}$ & $1.79 \pm 0.17$ & $1.87 \pm 0.18$ & 0.033 \\
\hline Body mass index, kg/m² & $25.3 \pm 3.1$ & $27.4 \pm 3.1$ & 0.002 \\
\hline \multicolumn{4}{|l|}{ Medical history, $n(\%)$} \\
\hline Hypertension & $23(47)$ & 27 (68) & 0.052 \\
\hline Diabetes mellitus & $14(29)$ & $14(35)$ & 0.516 \\
\hline Dyslipidemia & $40(82)$ & $39(98)$ & 0.043 \\
\hline \multicolumn{4}{|l|}{ Medication, $n$ (\%) } \\
\hline Platelet inhibitors & $34(69)$ & $37(93)$ & 0.007 \\
\hline Beta-Blockers & $25(51)$ & $33(83)$ & 0.002 \\
\hline Calcium channel blockers & $22(45)$ & $15(38)$ & 0.481 \\
\hline ACE inhibitors or ARBs & $11(22)$ & $20(50)$ & 0.007 \\
\hline Statins & $42(86)$ & 38 (95) & 0.275 \\
\hline \multicolumn{4}{|l|}{ Coronary angiography, $n(\%)$} \\
\hline One-Vessel disease & - & $27(68)$ & - \\
\hline Two-Vessel disease & - & $9(23)$ & - \\
\hline Three-Vessel disease & - & $4(10)$ & - \\
\hline Left main coronary artery & - & $2(5)$ & - \\
\hline Left anterior descending artery & - & $29(73)$ & - \\
\hline Left circumflex artery & - & $10(25)$ & - \\
\hline Right coronary artery & - & $16(40)$ & - \\
\hline \multicolumn{4}{|l|}{ Coronary dominance, $n(\%)$} \\
\hline Right coronary dominance & $45(92)$ & $36(90)$ & $>0.999$ \\
\hline Left coronary dominance & $3(6)$ & $3(8)$ & $>0.999$ \\
\hline Codominance & $1(2)$ & $1(3)$ & $>0.999$ \\
\hline ECG positive, $n(\%)$ & $15(31)$ & $7(18)$ & 0.154 \\
\hline \multicolumn{4}{|c|}{ Echocardiographic parameters } \\
\hline LVDd, mm & $47 \pm 3$ & $48 \pm 5$ & 0.239 \\
\hline LVDs, mm & $32 \pm 3$ & $31 \pm 3$ & 0.325 \\
\hline IVSd, mm & $9 \pm 1$ & $9 \pm 1$ & 0.338 \\
\hline LVPWd, mm & $8 \pm 1$ & $9 \pm 1$ & 0.016 \\
\hline LV mass index, $\mathrm{g} / \mathrm{m}^{2}$ & $78 \pm 16$ & $82 \pm 15$ & 0.173 \\
\hline Mitral E, m/s & $0.8 \pm 0.2$ & $0.8 \pm 0.2$ & 0.509 \\
\hline Mitral A, m/s & $0.8 \pm 0.2$ & $0.8 \pm 0.1$ & 0.531 \\
\hline Mitral E/A & $1.0 \pm 0.3$ & $1.0 \pm 0.3$ & 0.683 \\
\hline
\end{tabular}

$A C E$, angiotensin-converting enzyme; $A R B$, angiotensin II receptor blocker; $C A D$, coronary artery disease; $E C G$, electrocardiography; $L V$, left ventricular; $L V D d, L V$ enddiastolic diameter; LVDs, LV end-systolic diameter; LVPWd, LV posterior wall thickness in end-diastole; Mitral E, mitral early-diastole velocity; Mitral A, mitral late-diastole velocity; IVSd, interventricular septum thickness in end-diastole.

Data are expressed as mean $\pm S D$ or as number (percentage).

\section{Coronary Angiography}

The images from CAG were interpreted visually by an experienced cardiologist blinded to patients' echocardiographic data. Significant CAD was defined as $\geq 70 \%$ luminal diameter narrowing in one or more major epicardial vessels or $\geq 50 \%$ in the left main coronary (19).
TABLE 2 | Hemodynamic and echocardiographic parameters at rest and during treadmill exercise stress.

\begin{tabular}{|c|c|c|c|}
\hline Variable & $\begin{array}{l}\text { Non-Significant } \\
\text { CAD }(n=49)\end{array}$ & $\begin{array}{l}\text { Significant CAD } \\
(n=40)\end{array}$ & $P$-Value \\
\hline \multicolumn{4}{|l|}{ Rest } \\
\hline Heat rate, beats/min & $77 \pm 15$ & $75 \pm 13$ & 0.523 \\
\hline SBP, mm Hg & $132 \pm 20$ & $131 \pm 18$ & 0.914 \\
\hline DBP, $\mathrm{mm} \mathrm{Hg}$ & $78 \pm 12$ & $79 \pm 12$ & 0.581 \\
\hline LVEF, \% & $64 \pm 5$ & $62 \pm 4$ & 0.203 \\
\hline Rest GLS(AFI), \% & $-19.6 \pm 2.3$ & $-19.2 \pm 1.8$ & 0.323 \\
\hline Rest endocardial GLS, \% & $-24.0 \pm 3.0$ & $-23.9 \pm 2.6$ & 0.867 \\
\hline Rest mid-myocardial GLS, \% & $-21.0 \pm 2.9$ & $-20.9 \pm 2.3$ & 0.853 \\
\hline Rest epicardial GLS, \% & $-18.5 \pm 2.8$ & $-18.4 \pm 2.1$ & 0.825 \\
\hline Rest GWE, \% & $95(93,96)$ & $94(92,96)$ & 0.060 \\
\hline \multicolumn{4}{|l|}{ Peak exercise } \\
\hline Heat rate, beats/min & $140 \pm 8$ & $138 \pm 7$ & 0.161 \\
\hline SBP, mmHg & $176 \pm 20$ & $171 \pm 22$ & 0.268 \\
\hline $\mathrm{DBP}, \mathrm{mmHg}$ & $77 \pm 14$ & $77 \pm 15$ & 0.966 \\
\hline LVEF, \% & $67 \pm 3$ & $66 \pm 4$ & 0.180 \\
\hline Peak WMSI & $1.00(1.00,1.00)$ & $1.00(1.00,1.06)$ & 0.079 \\
\hline Peak GLS(AFI), \% & $-21.5 \pm 2.3$ & $-20.0 \pm 1.9$ & 0.001 \\
\hline Peak endocardial GLS, \% & $-28.8 \pm 2.7$ & $-26.0 \pm 3.0$ & $<0.001$ \\
\hline Peak mid-myocardial GLS, \% & $-25.0 \pm 2.4$ & $-22.8 \pm 2.7$ & $<0.001$ \\
\hline Peak epicardial GLS, \% & $-21.8 \pm 2.3$ & $-19.9 \pm 2.4$ & $<0.001$ \\
\hline$\Delta$ Endocardial GLS, \% & $-4.86 \pm 3.00$ & $-2.17 \pm 3.49$ & $<0.001$ \\
\hline$\Delta$ Mid-myocardial GLS, \% & $-4.02 \pm 2.72$ & $-1.90 \pm 3.12$ & 0.001 \\
\hline$\Delta$ Epicardial GLS, \% & $-3.30 \pm 2.67$ & $-1.57 \pm 2.84$ & 0.004 \\
\hline Peak GWE, \% & $94(93,95)$ & $90(87,93)$ & $<0.001$ \\
\hline \multicolumn{4}{|l|}{ Recovery } \\
\hline Heat rate, beats/min & $84 \pm 12$ & $83 \pm 10$ & 0.564 \\
\hline $\mathrm{SBP}, \mathrm{mmHg}$ & $155 \pm 22$ & $159 \pm 26$ & 0.409 \\
\hline DBP, mmHg & $69 \pm 11$ & $72 \pm 16$ & 0.377 \\
\hline LVEF, \% & $67 \pm 5$ & $66 \pm 6$ & 0.354 \\
\hline Recovery WMSI & $1.00(1.00,1.03)$ & $1.00(1.00,1.06)$ & 0.702 \\
\hline Recovery GLS(AFI), \% & $-21.4 \pm 2.7$ & $-20.7 \pm 2.0$ & 0.195 \\
\hline Recovery endocardial GLS, \% & $-27.0 \pm 3.5$ & $-26.9 \pm 2.8$ & 0.855 \\
\hline Recovery mid-myocardial GLS, & $\%-23.4 \pm 3.0$ & $-23.6 \pm 2.6$ & 0.841 \\
\hline Recovery epicardial GLS, \% & $-20.4 \pm 2.5$ & $-20.8 \pm 2.4$ & 0.562 \\
\hline Recovery GWE, \% & $95(94,96)$ & $93(91,95)$ & 0.001 \\
\hline
\end{tabular}

$A F I$, Automated function imaging; $C A D$, coronary artery disease; $D B P$, diastolic blood pressure; EF, ejection fraction; GLS, global longitudinal strain; GWE, global myocardial work efficiency; LV, left ventricular; SBP, systolic blood pressure; WMSI, wall motion score index.

$\Delta$-value, stress value-rest value. Data are expressed as mean $\pm S D$ or as median (interquartile range).

\section{Intra- and Interobserver Variability for Myocardial Layer-Specific Strain and GWE}

Intra- and interobserver variabilities were determined with 15 randomly selected patients, where the analysis was repeated by the same investigator 2 weeks later and by a second investigator blinded to the initial results of the first investigator, respectively. 


\section{Statistical Analysis}

Normally distributed continuous variables are presented as mean values \pm standard deviation or as the median value (interquartile range) when not normally distributed. Categorical variables are presented as absolute numbers and percentages. Normal distribution was verified by the ShapiroWilk test. Continuous variables were compared using Student's $t$-test or Mann-Whitney $U$-test, as appropriate, whereas categorical variables were compared with the chi-squared test or Fisher's exact test. Correlation between continuous variables was performed using Pearson's or Spearman's correlation coefficient. Univariate binary logistic regression analyses were performed to evaluate the association between the presence of significant $\mathrm{CAD}$ and the echocardiographic variables. After excluding variables that showed collinearity (Pearson's or Spearman's correlation coefficient $\geq 0.60$ or variance inflation factor $>10$ ), all variables that found to be significant on univariate binary logistic regression were considered for multiple binary regression analysis using the forward selection method. Receiver operating characteristic curve analysis and Youden's index were used to assess the optimal cutoff points for layer-specific strain and GWE in predicting significant CAD. The comparisons of AUCs were performed using the method described by DeLong et al. (20). Intraclass correlation coefficients and coefficients of variation were calculated during the inter- and intraobserver variability tests to evaluate the reproducibility.

Analyses were performed using SPSS, version 25.0 (IBM Corp., Armonk, NJ, USA), MedCalc, version 18.2.1 (MedCalc Software, Ltd., Ostend, Belgium), and GraphPad Prism 8 (GraphPad Software, San Diego, CA, USA). All statistical tests were two-sided, and a $p<0.05$ was considered statistically significant.

\section{RESULTS}

\section{Clinical Characteristics}

Of the 89 patients, 40 and 49 patients were diagnosed with significant and non-significant CAD by CAG. The demographic information, angiographic results, and exercise ECG results of both groups are presented in Table 1, and hemodynamic data during treadmill exercise testing are shown in Table 2. No statistically significant differences were observed (all $p>$ 0.05 ) between the two groups regarding age, gender, heart rate, blood pressure, coronary dominance, and exercise ECG results. Patients with significant CAD showed a larger body surface area and body mass index (both $p<0.05$ ) and a higher incidence of dyslipidemia $(p<0.05)$ compared with those in the nonsignificant CAD group (Table 1).

\section{Conventional Echocardiography}

No significant differences were observed (all $p>0.05$ ) between the two groups for the conventional echocardiographic parameters of LV end-diastolic or end-systolic dimensions, LV septal thickness in end-diastole, LV mass index, mitral earlydiastole velocity, mitral late-diastole velocity, and the ratio of mitral early-diastole velocity to late-diastole velocity. LV posterior wall thickness in end-diastole was significantly larger in the significant CAD group than that of the non-significant CAD group $(p<0.05$; Table 1$)$.

\section{GLS and Myocardial Layer-Specific Strain Analysis}

The LV GLS (AFI) had significantly lower absolute values during peak exercise in the significant CAD group $(p=0.001)$ compared with those in the non-significant group, although there were no significant differences observed during the rest or recovery periods (all $p>0.05$; Table 2).

Patients with significant CAD had a significantly worse function than those with non-significant CAD at peak exercise in all three myocardial layers when assessed with GLS (all $p<$ 0.001 ), although the differences between the two groups were not significant at rest or during recovery ( $p>0.05$; Figure 2, Table 2). The difference ( $\Delta$-value) in endocardial, mid-myocardial, and epicardial GLS from rest to peak exercise were, respectively significantly smaller in the significant $C A D$ group than that of patients with non-significant CAD (all $p<0.01$; Table 2).

\section{Global LV Myocardial Work Efficiency Analysis}

The GWE value was minimal during peak exercise and increased in the recovery period (Figure 2) in both groups. At peak exercise and recovery period, the values of GWE in patients with significant CAD were significantly lower than those of the non-significant CAD group (all $p<0.01$ ), although at rest, the values of the two groups had no significant differences $(p>0.05$; Table 2).

\section{Correlation Between GWE and Myocardial Strain at Peak Exercise}

Peak GWE showed modest negative correlations with peak endocardial, mid-myocardial, and epicardial GLS ( $r=-0.44$, -0.43 , and -0.43 , respectively; all $p<0.001)$ and a good negative correlation with peak GLS using AFI $(r=-0.63, p<0.001$; Supplementary Figure 1). Peak endocardial, mid-myocardial, and epicardial GLS $(r=0.46,0.47$, and 0.45 , respectively, all $p<0.001$ ) all presented modest positive correlations with peak GLS using AFI (Supplementary Figure 2). Peak endocardial GLS showed great correlations with both peak mid-myocardial GLS and peak epicardial GLS ( $r=0.98$, and 0.93, respectively, both $p<0.001)$.

\section{Binary Logistic Regression Analyses for the Detection of Significant CAD}

In univariable binary logistic regression analysis, body surface area, body mass index, dyslipidemia, LV posterior wall thickness in end-diastole, peak endocardial, mid-myocardial, epicardial GLS, peak GLS (AFI), peak GWE, and recovery GWE were significantly associated with significant CAD (Table 3).

In multivariable binary logistic regression analysis, peak endocardial GLS (odds ratio: 1.35, $p=0.006$ ) and peak GWE (odds ratio: $0.76, p<0.001$ ) were significantly associated with significant CAD (Table 3). 
A

\section{Endocardial GLS}

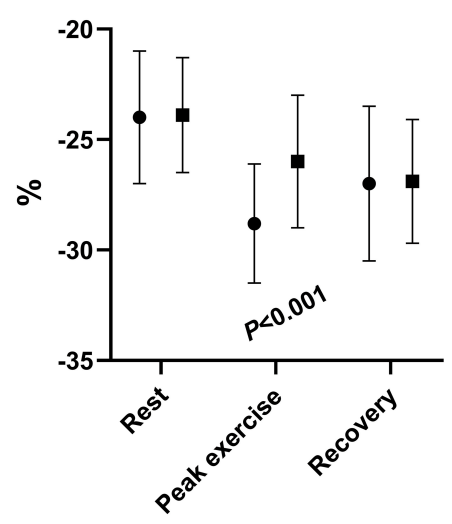

C

Epicardial GLS

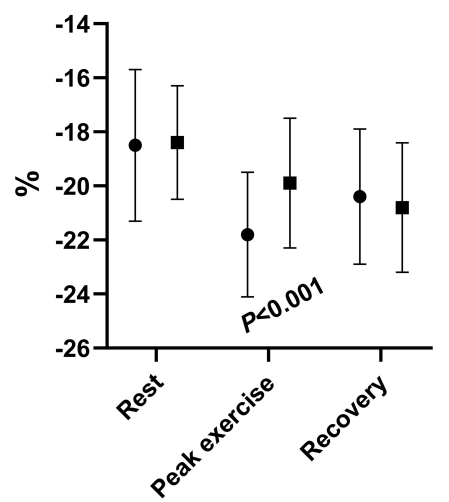

- Nonsignificant CAD

- Significant CAD
B

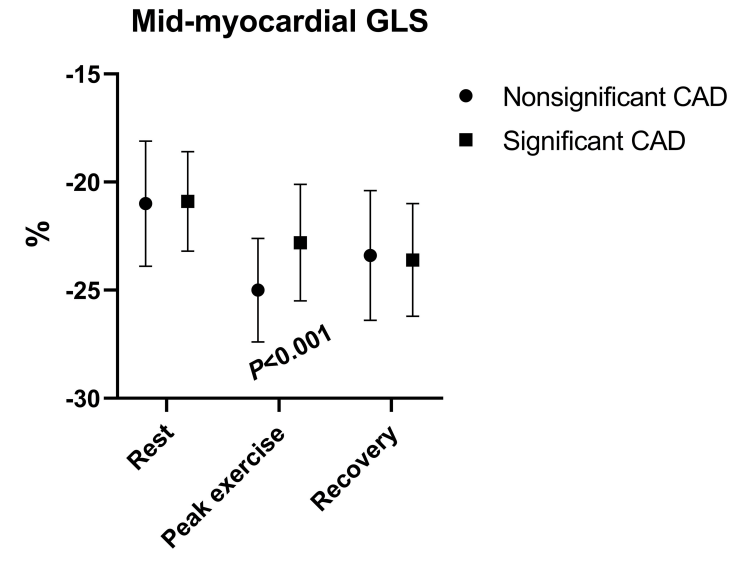

D

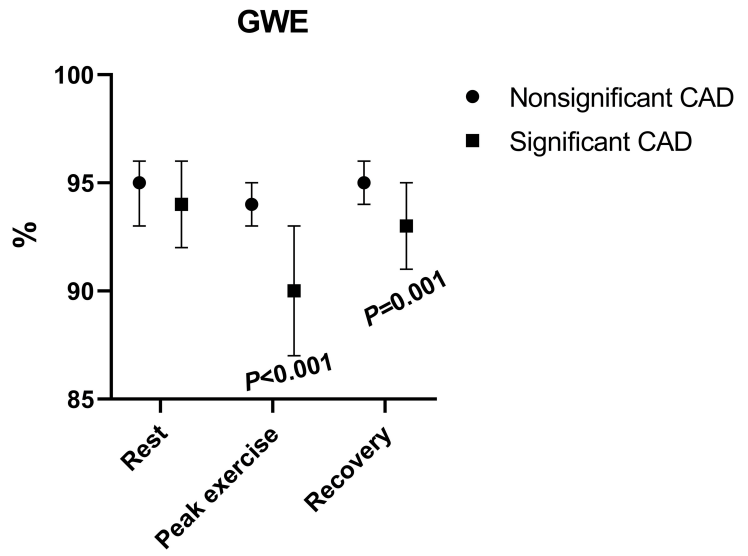

FIGURE 2 | The changes in myocardial longitudinal layer-specific strain [(A) endocardial GLS; (B) mid-myocardial GLS; (C) and epicardial GLS] and global myocardial work efficiency (D) between patients with nonsignificant CAD and significant CAD during rest, peak exercise, and recovery period. CAD, coronary artery disease; GLS, global longitudinal strain; GWE, global myocardial work efficiency.

TABLE 3 | Univariate and multivariate binary logistic regression analyses for detection of significant CAD.

\begin{tabular}{lccc}
\hline Parameters & \multicolumn{2}{c}{ Univariate binary logistic regression } & Multivariate binary logistic regression \\
\cline { 2 - 3 } & OR [95\% Cl] & $\boldsymbol{p}$-value & OR [95\% Cl] \\
\hline Body surface area, $\mathrm{m}^{2}$ & $14.97[1.19-189.02]$ & 0.036 & \\
Body mass index, kg/malue & 0.004 & \\
Dyslipidemia & $1.24[1.07-1.44]$ & 0.044 & \\
LVPWd, mm & $8.78[1.06-72.56]$ & 0.021 & $1.35[1.09-1.67]$ \\
Peak endocardial GLS, \% & $1.57[1.07-2.30]$ & $<0.001$ & \\
Peak mid-myocardial GLS, \% & $1.44[1.19-1.75]$ & 0.001 & \\
Peak epicardial GLS, \% & $1.44[1.18-1.78]$ & 0.001 & 0.006 \\
Peak GLS(AFI), \% & $1.43[1.15-1.77]$ & 0.002 & $0.76[0.64-0.90]$ \\
Peak GWE, \% & $1.39[1.13-1.72]$ & $<0.001$ & \\
Recovery GWE, \% & $0.71[0.60-0.84]$ & 0.013 & 0.001
\end{tabular}

Cl, confidence interval; OR, odds ratio; for other abbreviations see Tables 1, 2. 

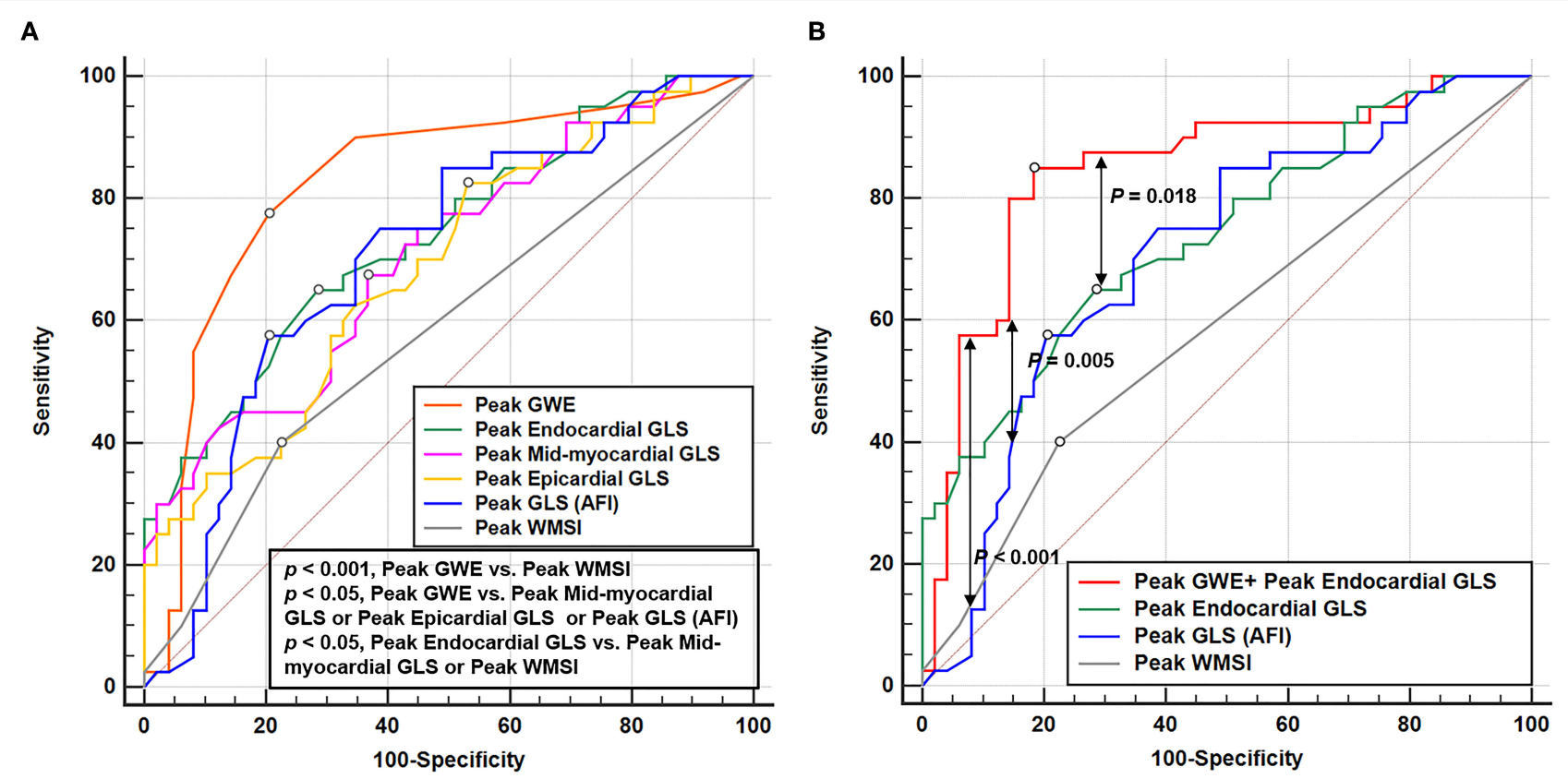

FIGURE 3 | Receiver operating characteristic curves of echocardiographic parameters at peak exercise to detect the significant CAD. (A) Receiver operating characteristic curves of GWE, layer-specific strain, GLS (AFI), and WMSI at peak exercise in identifying significant CAD. (B) Receiver operating characteristic curve of the parameter combined peak GWE and peak endocardial GLS, peak endocardial GLS, peak GLS (AFI), and peak WMSI in detecting significant CAD. CAD, coronary artery disease; GLS, global longitudinal strain; GWE, global myocardial work efficiency; AFI, automated function imaging; WMSI, wall motion score index.

TABLE 4 | Receiver operating characteristic curve analysis for the detection of significant CAD.

\begin{tabular}{|c|c|c|c|c|c|c|c|}
\hline Parameters & AUC & $95 \% \mathrm{Cl}$ & $p$-value & Cutoff value & Sensitivity (\%) & Specificity (\%) & Youden's index \\
\hline Peak GWE + Peak endocardial GLS & 0.848 & $0.756-0.915$ & $<0.001$ & $>0.406$ & 85.0 & 81.6 & 0.666 \\
\hline Peak GWE, \% & 0.827 & $0.733-0.899$ & $<0.001$ & $\leq 92$ & 77.5 & 79.6 & 0.571 \\
\hline Peak endocardial GLS, \% & 0.739 & $0.635-0.827$ & $<0.001$ & $>-27.0$ & 65.0 & 71.4 & 0.364 \\
\hline Peak mid-myocardial GLS, \% & 0.708 & $0.602-0.800$ & $<0.001$ & $>-24.0$ & 67.5 & 63.3 & 0.308 \\
\hline Peak epicardial GLS, \% & 0.688 & $0.581-0.782$ & 0.001 & $>-21.8$ & 82.5 & 46.9 & 0.294 \\
\hline Peak GLS (AFI), \% & 0.708 & $0.603-0.800$ & $<0.001$ & $>-20.0$ & 57.5 & 79.6 & 0.371 \\
\hline Peak WMSI & 0.587 & $0.478-0.691$ & 0.080 & $>1.00$ & 40.0 & 77.6 & 0.176 \\
\hline$\Delta$ Endocardial GLS, $\%$ & 0.721 & $0.616-0.811$ & $<0.001$ & $>-3.6$ & 67.5 & 71.4 & 0.389 \\
\hline$\Delta$ Mid-myocardial GLS, \% & 0.702 & $0.596-0.795$ & $<0.001$ & $>-1.5$ & 50.0 & 85.7 & 0.357 \\
\hline$\Delta$ Epicardial GLS, \% & 0.684 & $0.577-0.778$ & 0.001 & $>-4.4$ & 85.0 & 49.0 & 0.340 \\
\hline
\end{tabular}

AUC, area under the curve; Cl, confidence interval; $\Delta$-value, stress value-rest value; for other abbreviations see as Tables $\mathbf{1}, \mathbf{2}$.

\section{Receiver Operating Characteristic Curve Analysis for the Detection of Significant CAD}

According to receiver operating characteristic curve analysis, the cutoff value for the best possible detection of peak GWE in the significant CAD group was 92\%. Peak GWE had the highest area under the curve (AUC) for the detection of significant CAD (AUC, 0.827; $p<0.001$ ) that was superior to peak mid-myocardial and epicardial GLS (AUC, 0.708, and 0.688, respectively, both $p<0.05$; Figure $3 \mathrm{~A}$, Table 4), although it was not statistically better than endocardial GLS (AUC: 0.739, $p>0.05)$. However, the addition of peak GWE to peak endocardial GLS significantly increased the AUC over that of peak endocardial GLS (AUC: 0.848 vs. 0.739, $p=0.018$; Figure 3B, Table 4). The AUCs of the combination of peak GWE and peak endocardial GLS or peak GWE alone were significantly higher than that of peak GLS using AFI (Figures 3A,B, Table 4). The AUC of peak GLS (AFI) was similar to those of peak myocardial layer-specific GLS (0.708 vs. endocardial, 0.739; midmyocardial, 0.708, and epicardial, 0.688; all $p>0.05$; Figure 3A, Table 4). The AUCs of peak myocardial layer-specific GLS and peak GWE were better than peak WMSI, which represented the conventional exercise stress echocardiography (Figure 3A, Table 4). The $\Delta$-value of myocardial layer-specific GLS also had 
high AUCs to identify significant CAD (endocardial, 0.721; midmyocardial, 0.702; and epicardial, 0.684; Table 4).

\section{Intra- and Interobserver Variabilities for Layer-Specific Strain Parameters and GWE} The intra- and interobserver variabilities for peak myocardial layer-specific GLS and peak GWE, which showed good repeatability and reproducibility, are summarized in Supplementary Table 1.

\section{DISCUSSION}

This is the first study to compare the ability of myocardial layer-specific strain and GWE in identifying significant CAD in patients with angina pectoris without a known history of CAD. The primary findings were that both myocardial layer-specific GLS and GWE at peak exercise can discriminate significant CAD. At peak exercise, GWE was superior to myocardial layerspecific GLS as a non-invasive measure for the detection of significant CAD. Furthermore, the combination of peak GWE and peak endocardial GLS had incremental diagnostic value for the detection of significant CAD when compared to that of myocardial layer-specific GLS at peak exercise alone.

\section{Myocardial Layer-Specific Strain for the Detection of Significant CAD Compared With GLS Based on AFI or WMSI During Exercise Stress}

Previous studies have reported excellent intra- and interobserver reproducibilities of layer-specific strain $(21,22)$. In addition, studies have also investigated the diagnostic value of layerspecific strain on stress echocardiography in patients suspected of CAD (23-26). Nishi et al. suggested that layer-specific GLS at the early recovery phase after cycle ergometer exercise stress was significantly more impaired in the ischemic territories than in non-ischemic territories (26). However, in our research, the differences in layer-specific strain during the rest or recovery periods between significant and non-significant CAD groups were less pronounced. Only layer-specific strain at peak exercise after treadmill exercise stress testing provided a high value for the non-invasive identification of significant CAD. Despite the statement of the stress period is different, the definition of the early recovery period in the study of Nishi et al. was similar to the definition of peak exercise in our research (from immediate cessation to $<3 \mathrm{~min}$ after exercise vs. $<1 \mathrm{~min}$ after treadmill exercise), and the definition of the recovery period in our research was $>3$ min after treadmill exercise. Therefore, the results are consistent.

The LV heart wall comprises three layers: the oblique endocardial, the circular mid-myocardial, and the oblique epicardial layer. Since the endocardial layer of the myocardium is more susceptible to ischemia than the epicardial layer, and the endocardial-layer fibers are mainly oriented in the longitudinal direction $(27,28)$, it is likely to expect in patients with CAD that ischemia extends from endocardium to epicardium and endocardial GLS deteriorates before epicardial GLS abnormalities become apparent (29). However, the previous studies did not demonstrate clear superiority for endocardiallayer strain over other layers on stress echocardiography for the detection of significant CAD $(23,26)$. Nishi et al. found that the diagnostic ability of the endocardial, mid-myocardial, and epicardial GLS at the early recovery phase on cycle ergometer exercise stress echocardiography (AUC; 0.621, 0.619, and 0.616, respectively), are comparable (26), which was also found by Ejlersen et al. (23). Similarly, in this study, the myocardial layer-specific GLS analysis revealed that all myocardial layers were affected in patients with significant CAD at peak exercise. However, the AUCs for the detection of significant CAD were not statistically different among the three layers at peak exercise although the AUC of endocardial GLS was the highest among all three layers.

In line with previous research $(23,26,30)$, myocardial layerspecific GLS was better than the semiquantitative method of WMSI in stress echocardiography to identify significant CAD. Besides, with previous studies demonstrated that GLS added value to exercise stress testing to diagnose various cardiac diseases, including myocardial ischemia $(30,31)$. We also compared the diagnostic value of layer-specific GLS and GLS (AFI) during exercise stress to identify significant CAD and found that myocardial layer-specific GLS was relative to GLS (AFI). However, no statistical differences were observed in the diagnostic value of the three layer-specific GLS and GLS (AFI) when applied to treadmill exercise stress echocardiography, although the AUC of endocardial GLS was better than that of GLS (AFI), which were consistent with previous studies $(23,25)$. Therefore, it was confirmed that endocardial GLS at peak exercise was superior to the conventional stress echocardiography and has no significant superiority over GLS (AFI) or other layer-specific strain in identifying myocardial ischemia.

In addition, we also explored the difference in endocardial, mid-myocardial, and epicardial GLS from rest to peak exercise and found that significant CAD can be effectively identified. The diagnostic value of the differences in endocardial, midmyocardial, and epicardial GLS from rest to peak exercise was comparable to endocardial, mid-myocardial, and epicardial GLS at peak exercise.

\section{Incremental Diagnostic Value of GWE}

The myocardial layer-specific GLS had a high diagnostic value for significant CAD, but these parameters were still limited by load dependency. An increase in afterload would lead to a reduction in strain, which may underestimate the true myocardial contractility, especially in the context of stress echocardiography (32). MW can overcome this limitation and reflect the true contractility of the myocardium by establishing the GWE, which combines the global constructive and wasted work of the myocardium, which can better reflect the myocardial changes affected by ischemia.

Recently, various studies have proved the diagnostic and prognostic values of GWE (6-12). However, the comparison of the diagnostic capability of GWE and myocardial layer-specific GLS during peak exercise in identifying significant CAD in patients without a history of CAD has not been explored prior to our study. We observed that the GWE was moderately correlated with the myocardial layer-specific GLS at peak exercise but 
stronger with the GLS obtained by AFI, because MW derived from GLS (based on AFI) and brachial artery blood pressure. The peak GWE was superior in the detection of significant CAD when compared to that of the WMSI, GLS (AFI), mid-myocardial, and epicardial GLS at peak. However, the diagnostic capability of peak GWE did not show statistically significant superiority over peak endocardial GLS, although the AUC of peak GWE was higher than that of peak endocardial GLS. The combination of peak GWE and peak endocardial GLS could significantly improve the discrimination ability for significant CAD when compared to endocardial GLS alone. The present research extends the usefulness of peak GWE to patients with CAD with normal LV ejection fraction and baseline wall motion and suggests an incremental diagnostic value of peak GWE over the myocardial layer-specific GLS in patients whose rest echocardiography does not suggest CAD. The MW can only indicate overall-layer myocardial function but could not reflect each layer's MW at present, which can be explored in future studies.

\section{Clinical Implications}

In patients with angina pectoris and no history of CAD, conventional echocardiography during exercise stress was not accurate enough to detect significant CAD. The use of GWE or myocardial layer-specific GLS at peak exercise, especially the combination of peak GWE and peak endocardial GLS, provided a better non-invasive screening method to identify significant $\mathrm{CAD}$ before radioactive or invasive exams. Peak GWE is particularly important in measurements performed during exercise stress testing, or in patients with inadequate blood pressure control. However, the practical applicability of these new parameters to assist the decisionmaking process for significant $\mathrm{CAD}$ needs to be verified in further studies.

\section{LIMITATIONS}

This was a retrospective, single-center study, and prospective validation is needed. Selection bias inherent in any study comparing stress echocardiography with invasive angiography may have occurred in our research since only patients with chest pain who underwent invasive angiography were included. The circumferential and radial strains were not investigated in this study, since the myocardial fibers most vulnerable to ischemia are the longitudinally orientated fibers that are located subendocardial, and longitudinal strain is thought to be the most sensitive parameter to detect CAD (33). Since LV GWE is predicated on the measurement of GLS, it is not a vendor-independent measure and is still influenced by the image quality. Fractional flow reserve measurements were not taken in this study, and therefore, the true hemodynamic relevance of the stenoses is not known. Despite this limitation, the fraction of patients with non-flow limiting stenosis may have been reduced since a high cutoff for significant CAD was used.

\section{CONCLUSION}

In patients with angina pectoris and normal baseline wall motion, both peak GWE and peak endocardial GLS are independent predictors of significant CAD that can significantly improve the diagnostic performance of exercise stress echocardiography. Furthermore, adding peak GWE to peak endocardial GLS provides incremental diagnostic value in non-invasive screening for significant $\mathrm{CAD}$ before radioactive or invasive examinations.

\section{DATA AVAILABILITY STATEMENT}

The raw data supporting the conclusions of this article will be made available by the authors, without undue reservation.

\section{ETHICS STATEMENT}

The studies involving human participants were reviewed and approved by Ethics Committees of Fuwai Hospital (No. 20181121). The participants or their legal representatives provided their written informed consent to participate in this study.

\section{AUTHOR CONTRIBUTIONS}

WW, ZZ, and HW contributed to the conception, design, supervision of the study, and revised the manuscript critically for intellectual content. JL drafted the manuscript. LG, JH, LN, YZ, XL, and JW contributed to the collection and interpretation of data. JL, ML, and YC contributed to statistical analysis. All authors contributed to the article and approved the submitted version.

\section{FUNDING}

This study was supported by the Beijing Municipal Science and Technology Commission (Grant No. Z171100001017213) and Construction Research Project of Key Laboratory (Cultivation) of Chinese Academy of Medical Sciences (Grant No. 2019PT310025).

\section{ACKNOWLEDGMENTS}

We sincerely thank the colleagues of the Department of Echocardiography, Cardiology, and the Functional Testing Center of Fuwai Hospital for their effort and assistance. We would like to thank Editage (www.editage.cn) for English language editing.

\section{SUPPLEMENTARY MATERIAL}

The Supplementary Material for this article can be found online at: https://www.frontiersin.org/articles/10.3389/fcvm. 2021.786943/full\#supplementary-material

Supplementary Figure 1 | Correlation between GWE and layer-specific strain, GLS (AFI) at peak exercise. (A) Correlation between peak GWE and peak endocardial GLS. (B) Correlation between peak GWE and peak mid-myocardial 
GLS. (C) Correlation between peak GWE and peak epicardial GLS. (D) Correlation between peak GWE and peak GLS (AFI). GLS, global longitudinal strain; GWE, global myocardial work efficiency; AFI, automated function imaging.

Supplementary Figure 2 | Correlation between layer-specific strain and GLS (AFI) at peak exercise. (A) Correlation between peak endocardial GLS and peak GLS (AFI). (B) Correlation between peak mid-myocardial GLS and peak GLS (AFI).
(C) Correlation between peak epicardial GLS and peak GLS (AFI). GLS, global longitudinal strain; AFI, automated function imaging.

Supplementary Table 1 | Intra- and inter-observer variabilities for peak layer-specific strain parameters and peak GWE. ICC, Intraclass correlation coefficient; $\mathrm{Cl}$, confidence interval; CV, coefficient of variation; GLS, global longitudinal strain; GWE, global myocardial work efficiency.

\section{REFERENCES}

1. Patel MR, Peterson ED, Dai D, Brennan JM, Redberg RF, Anderson HV, et al. Low diagnostic yield of elective coronary angiography. N Engl J Med. (2010) 362:886-95. doi: 10.1056/NEJMoa0907272

2. Task Force M, Montalescot G, Sechtem U, Achenbach S, Andreotti F, Arden C, et al. 2013 ESC guidelines on the management of stable coronary artery disease: the task force on the management of stable coronary artery disease of the European society of cardiology. Eur Heart J. (2013) 34:29493003. doi: 10.1093/eurheartj/eht 296

3. Ilardi F, Marchetta S, Martinez C, Sprynger M, Ancion A, Manganaro R, et al. Impact of aortic stenosis on layer-specific longitudinal strain: relationship with symptoms and outcome. Eur Heart J Cardiovasc Imaging. (2020) 21:40816. doi: $10.1093 /$ ehjci/jez215

4. Huttin O, Girerd N, Coiro S, Bozec E, Selton-Suty C, Lamiral Z, et al. Association between layer-specific longitudinal strain and risk factors of heart failure and dyspnea: a population-based study. J Am Soc Echocardiogr. (2019) 32:854-65.e8. doi: 10.1016/j.echo.2019.03.011

5. Sarvari SI, Haugaa KH, Zahid W, Bendz B, Aakhus S, Aaberge L, et al. Layer-specific quantification of myocardial deformation by strain echocardiography may reveal significant CAD in patients with non-STsegment elevation acute coronary syndrome. JACC Cardiovasc Imaging. (2013) 6:535-44. doi: 10.1016/j.jcmg.2013.01.009

6. Minhas AS, Gilotra NA, Goerlich E, Metkus T, Garibaldi BT, Sharma G, et al. Myocardial work efficiency, a novel measure of myocardial dysfunction, is reduced in COVID-19 patients and associated with in-hospital mortality. Front Cardiovasc Med. (2021) 8:667721. doi: 10.3389/fcvm.2021.667721

7. Lin J, Wu W, Gao L, He J, Zhu Z, Pang K, et al. Global myocardial work combined with treadmill exercise stress to detect significant coronary artery disease. J Am Soc Echocardiogr. (2021). doi: 10.1016/j.echo.2021.10.009. [Epub ahead of print].

8. Huang J, Yang C, Yan ZN, Fan L, Ni CF. Global myocardial work: a new way to detect subclinical myocardial dysfunction with normal left ventricle ejection fraction in essential hypertension patients: compared with myocardial layer-specific strain analysis. Echocardiography. (2021) 38:85060. doi: 10.1111/echo.15063

9. D'Andrea A, Ilardi F, D'Ascenzi F, Bandera F, Benfari G, Esposito $\mathrm{R}$, et al. Impaired myocardial work efficiency in heart failure with preserved ejection fraction. Eur Heart J Cardiovasc Imaging. (2021) 22:131220. doi: $10.1093 /$ ehjci/jeab153

10. El Mahdiui M, van der Bijl P, Abou R, Ajmone Marsan N, Delgado V, Bax JJ. Global left ventricular myocardial work efficiency in healthy individuals and patients with cardiovascular disease. $J$ Am Soc Echocardiogr. (2019) 32:1120-7. doi: 10.1016/j.echo.2019. 05.002

11. Lustosa RP, Butcher SC, van der Bijl P, El Mahdiui M, MonteroCabezas JM, Kostyukevich MV, et al. Global left ventricular myocardial work efficiency and long-term prognosis in patients after ST-segmentelevation myocardial infarction. Circ Cardiovasc Imaging. (2021) 14:e012072. doi: 10.1161/CIRCIMAGING.120.012072

12. van der Bijl P, Vo NM, Kostyukevich MV, Mertens B, Ajmone Marsan N, Delgado V, et al. Prognostic implications of global, left ventricular myocardial work efficiency before cardiac resynchronization therapy. Eur Heart J Cardiovasc Imaging. (2019) 20:1388-1394. doi: 10.1093/ehjci/jez095

13. Mitchell C, Rahko PS, Blauwet LA, Canaday B, Finstuen JA, Foster MC, et al. Guidelines for performing a comprehensive transthoracic echocardiographic examination in adults: recommendations from the

American society of echocardiography. J Am Soc Echocardiogr. (2019) 32:164. doi: 10.1016/j.echo.2018.06.004

14. Pellikka PA, Arruda-Olson A, Chaudhry FA, Chen MH, Marshall JE, Porter TR, et al. Guidelines for performance, interpretation, and application of stress echocardiography in ischemic heart disease: from the American society of echocardiography. J Am Soc Echocardiogr. (2020) 33:1-41 e8. doi: 10.1016/j.echo.2019.07.001

15. Lang RM, Badano LP, Mor-Avi V, Afilalo J, Armstrong A, Ernande L, et al. Recommendations for cardiac chamber quantification by echocardiography in adults: an update from the American society of echocardiography and the European association of cardiovascular imaging. J Am Soc Echocardiogr. (2015) 28:1-39.e14. doi: 10.1016/j.echo.2014.10.003

16. Eek C, Grenne B, Brunvand $\mathrm{H}$, Aakhus S, Endresen $\mathrm{K}$, Hol $\mathrm{PK}$, et al. Strain echocardiography and wall motion score index predicts final infarct size in patients with non-ST-segmentelevation myocardial infarction. Circ Cardiovasc Imaging. (2010) 3:187-94. doi: 10.1161/CIRCIMAGING.109.910521

17. Smiseth OA, Donal E, Penicka M, Sletten OJ. How to measure left ventricular myocardial work by pressure-strain loops. Eur Heart J Cardiovasc Imaging. (2021) 22:259-61. doi: 10.1093/ehjci/jeaa301

18. Russell K, Eriksen M, Aaberge L, Wilhelmsen N, Skulstad H, Remme EW, et al. A novel clinical method for quantification of regional left ventricular pressurestrain loop area: a non-invasive index of myocardial work. Eur Heart J. (2012) 33:724-33. doi: 10.1093/eurheartj/ehs016

19. Patel MR, Dehmer GJ, Hirshfeld JW, Smith PK, Spertus JA. ACCF/SCAI/STS/AATS/AHA/ASNC/HFSA/SCCT 2012 appropriate use criteria for coronary revascularization focused update: a report of the American college of cardiology foundation appropriate use criteria task force, society for cardiovascular angiography and interventions, society of thoracic surgeons, American association for thoracic surgery, American heart association, American society of nuclear cardiology, and the society of cardiovascular computed tomography. J Am Coll Cardiol. (2012) 59:857-81. doi: 10.1016/j.jtcvs.2012.01.061

20. DeLong ER, DeLong DM, Clarke-Pearson DL. Comparing the areas under two or more correlated receiver operating characteristic curves: a nonparametric approach. Biometrics. (1988) 44:837-45 doi: 10.2307/2531595

21. Ramlogan S, Aly D, France R, Schmidt S, Hinzman J, Sherman A, et al. Reproducibility and intervendor agreement of left ventricular global systolic strain in children using a layer-specific analysis. J Am Soc Echocardiogr. (2020) 33:110-9. doi: 10.1016/j.echo.2019.08.004

22. Coiro S, Huttin O, Bozec E, Selton-Suty C, Lamiral Z, Carluccio E, et al. Reproducibility of echocardiographic assessment of 2Dderived longitudinal strain parameters in a population-based study (the STANISLAS Cohort study). Int J Cardiovasc Imaging. (2017) 33:1361-9. doi: 10.1007/s10554-017-1117-z

23. Ejlersen JA, Poulsen SH, Mortensen J, May O. Diagnostic value of layerspecific global longitudinal strain during adenosine stress in patients suspected of coronary artery disease. Int J Cardiovasc Imaging. (2017) 33:47380. doi: $10.1007 / \mathrm{s} 10554-016-1022-\mathrm{x}$

24. Mandoli GE, Cameli M, Minardi S, Crudele F, Lunghetti S, Mondillo S. Layer-specific strain in dipyridamole stress echo: a new tool for the diagnosis of microvascular angina. Echocardiography. (2018) 35:200513. doi: $10.1111 /$ echo. 14180

25. Cadeddu Dessalvi C, Deidda M, Farci S, Longu G, Mercuro G. Early ischemia identification employing 2D speckle tracking selective layers analysis during dobutamine stress echocardiography. Echocardiography. (2019) 36:22028. doi: 10.1111/echo.14535 
26. Nishi T, Funabashi N, Ozawa K, Nishi T, Kamata T, Fujimoto $\mathrm{Y}$, et al. Regional layer-specific longitudinal peak systolic strain using exercise stress two-dimensional speckle-tracking echocardiography for the detection of functionally significant coronary artery disease. Heart Vessels. (2019) 34:1394403. doi: 10.1007/s00380-019-01361-w

27. Oh BH, Volpini M, Kambayashi M, Murata K, Rockman HA, Kassab GS, et al. Myocardial function and transmural blood flow during coronary venous retroperfusion in pigs. Circulation. (1992) 86:126579. doi: 10.1161/01.CIR.86.4.1265

28. Reimer KA, Lowe JE, Rasmussen MM, Jennings RB. The wavefront phenomenon of ischemic cell death. 1. Myocardial infarct size vs duration of coronary occlusion in dogs. Circulation. (1977) 56:78694. doi: 10.1161/01.CIR.56.5.786

29. Nishi T, Funabashi N, Ozawa K, Takahara M, Fujimoto Y, Kamata T, et al. Resting multilayer 2D speckle-tracking transthoracic echocardiography for the detection of clinically stable myocardial ischemic segments confirmed by invasive fractional flow reserve. Part 1: Vessel-by-vessel analysis. Int J Cardiol. (2016) 218:324-32. doi: 10.1016/j.ijcard.2016.05.016

30. Biering-Sorensen T, Hoffmann S, Mogelvang R, Zeeberg Iversen A, Galatius $\mathrm{S}$, Fritz-Hansen $\mathrm{T}$, et al. Myocardial strain analysis by 2-dimensional speckle tracking echocardiography improves diagnostics of coronary artery stenosis in stable angina pectoris. Circ Cardiovasc Imaging. (2014) 7:5865. doi: 10.1161/CIRCIMAGING.113.000989

31. Simonovic D, Coiro S, Deljanin-Ilic M, Kobayashi M, Carluccio E, Girerd $\mathrm{N}$, et al. Exercise-induced B-lines in heart failure with preserved ejection fraction occur along with diastolic function worsening. ESC Heart Fail. (2021) 8:5068-80. doi: 10.1002/ehf2.13575
32. Boe E, Russell K, Eek C, Eriksen M, Remme EW, Smiseth OA, et al. Non-invasive myocardial work index identifies acute coronary occlusion in patients with non-ST-segment elevation-acute coronary syndrome. Eur Heart J Cardiovasc Imaging. (2015) 16:1247-55. doi: 10.1093/ehjci/jev078

33. Geyer H, Caracciolo G, Abe H, Wilansky S, Carerj S, Gentile F, et al. Assessment of myocardial mechanics using speckle tracking echocardiography: fundamentals and clinical applications. J Am Soc Echocardiogr. (2010) 23:351-69; quiz 453-5. doi: 10.1016/j.echo.2010.02.015

Conflict of Interest: The authors declare that the research was conducted in the absence of any commercial or financial relationships that could be construed as a potential conflict of interest.

Publisher's Note: All claims expressed in this article are solely those of the authors and do not necessarily represent those of their affiliated organizations, or those of the publisher, the editors and the reviewers. Any product that may be evaluated in this article, or claim that may be made by its manufacturer, is not guaranteed or endorsed by the publisher.

Copyright $\odot 2022$ Lin, Gao, He, Liu, Cai, Niu, Zhao, Li, Wang, Wu, Zhu and Wang. This is an open-access article distributed under the terms of the Creative Commons Attribution License (CC BY). The use, distribution or reproduction in other forums is permitted, provided the original author(s) and the copyright owner(s) are credited and that the original publication in this journal is cited, in accordance with accepted academic practice. No use, distribution or reproduction is permitted which does not comply with these terms. 